\title{
Suspected Metallic Embolism following Endovascular Treatment of Intracranial Aneurysms
}

\author{
(D) Yasuda, (D) Maeda, (D). Umino, (D). Nakatsuka, (D). Umeda, (D) N. Toma, (D) H. Sakaida, and (D). Suzuki
}

\begin{abstract}
SUMMARY: We describe a case series of suspected metallic embolism after coil embolization for intracranial aneurysms. Between January 2012 and December 2014, 110 intracranial aneurysms had been treated by coil embolization in our institution. In 6 cases, the postprocedural MR imaging revealed abnormal spotty lesions not detected on the preprocedural MR imaging. The lesions were also undetectable on the postprocedural CT scan. They were demonstrated as low-intensity spots on TIWI, T2WI, DWI, and T2*-weighted imaging. On DWI, they were accompanied by bright "halo," and on T2*-weighted imaging, they showed a "blooming" effect. In 3 of the 6 cases, follow-up MR imaging was available and all the lesions remained and demonstrated no signal changes. Although histologic examination had not been performed, these neuroradiologic findings strongly supported the lesions being from metallic fragments. No specific responsible device was detected after reviewing all the devices used for the neuroendovascular treatment in the 6 cases.
\end{abstract}

C linically asymptomatic emboli are a common complication of cerebral angiography and neuroendovascular procedures, occurring in approximately $20 \%$ of cases. ${ }^{1}$ Foreign body emboli after cerebral angiography are a much less known complication, and only a few isolated cases have been reported. ${ }^{2-4}$ Recently, we have noticed abnormal signals suggesting metallic emboli on the postprocedural MR imaging in several cases of coil embolization for cerebral aneurysms. In this report, we describe the particular MR imaging findings of suspected metallic emboli following neuroendovascular procedures and discuss possible sources for these findings.

\section{Case Series}

After the retrospective review of our medical data base between January 2012 and December 2014 that contains 110 intracranial aneurysms treated by coil embolization, we encountered 6 cases that showed abnormal spotty lesions on the postprocedural MR imaging. The details of the clinical features of the 6 cases are summarized in Table 1. The devices used for the treatment in the 6 cases are summarized in Table 2 . In all 6 cases, the lesions were low

Received December 27, 2015; accepted after revision March 3, 2016.

From the Departments of Neurosurgery (R.Y., Y.N., Y.U., N.T., H.Sakaida, H.Suzuki) and Radiology (M.U., M.M.), Mie University Graduate School of Medicine, Tsu, Mie, Japan.

Paper previously presented, in part, at: 44th Annual Meeting of the Chubu Society of Neuroendovascular Therapy, August 8, 2015; Fukui, Japan.

Please address correspondence to Ryuta Yasuda, MD, 2-174 Edobashi, Tsu, Mie, Japan 513-8507; e-mail: yasudar1@gmail.com

http://dx.doi.org/10.3174/ajnr.A4804 intensity on T1WI, T2WI, DWI, and T2*-weighted images. Additionally, they were accompanied by a hyperintense "halo" on DWI and showed a "blooming" effect on $\mathrm{T} 2{ }^{*}$-weighted images. In 5 of the 6 cases (cases 1-5), a postprocedural CT scan was obtained but none of the lesions were detected. Follow-up MR images were available in 3 of the 6 cases (cases 1, 5, and 6), and in all 3 cases, the lesions remained without any signal changes during the follow-up periods (range, 6-34 months). No patients showed any symptoms associated with the lesions.

\section{Illustrative Cases}

Case 1. A 68-year-old woman was treated for a left ICA aneurysm (Fig 1A) with coil embolization. On the preprocedural MR imaging, no abnormal findings apart the aneurysm were identified. For the coil embolization procedure, a $5 \mathrm{~F}$ guiding sheath was placed in the left ICA and a 1.7F microcatheter was inserted into the aneurysm by using a 0.014 -inch microguidewire. The aneurysm was embolized with multiple platinum coils (Fig $1 B$ ). The MR images were obtained the next day by using a $1.5 \mathrm{~T}$ MR imaging unit (Achieva Nova Dual; Philips Healthcare, Best, the Netherlands) with a 5-mm section thickness, and DWI revealed lowintensity spots accompanied by a hyperintense halo at the left medial frontal and left parietal regions (Fig $1 C,-D$ ). These signals were obviously different from the bright lesion in the left parietal lobe indicating thromboembolism (Fig $1 E$ ) that showed no signal changes on $\mathrm{T} 2^{\star}$-weighted images.

While these lesions were shown as low-intensity spots on T1WI (Fig 1F) and T2WI (Fig 1G), they had a blooming effect on 
Table 1: Patient characteristics

\begin{tabular}{lclllcc}
\hline Case No. & $\begin{array}{c}\text { Patient Age } \\
(\mathbf{y r}) / \text { Sex }\end{array}$ & \multicolumn{1}{c}{ Location of Aneurysm } & $\begin{array}{c}\text { Aneurysm Size } \\
(\mathbf{m m})\end{array}$ & Procedure & $\begin{array}{c}\text { Procedural Time } \\
\text { (min) }\end{array}$ & $\begin{array}{c}\text { Postprodedural } \\
\text { Symptoms }\end{array}$ \\
\hline 1 & $68 / \mathrm{F}$ & Left ICA & $10.2 \times 8.2 \times 7.3$ & Coil embolization & 170 & None \\
2 & $56 / \mathrm{M}$ & Right vertebral artery & $15.6 \times 7.1 \times 6.5$ & Internal trapping & 135 & None \\
3 & $80 / \mathrm{F}$ & Right ICA & $12.6 \times 10.3 \times 11.0$ & Coil embolization & 150 & None \\
4 & $45 / \mathrm{F}$ & Right ICA & $4.9 \times 4.5 \times 4.3$ & Coil embolization & 158 & None \\
5 & $70 / \mathrm{M}$ & Anterior communicating artery & $9.6 \times 7.1 \times 9.5$ & Coil embolization & 145 & None \\
6 & $41 / \mathrm{F}$ & Left ICA & $5.6 \times 4.9 \times 3.8$ & Coil embolization & 150 & None \\
\hline
\end{tabular}

Table 2: Devices used for treatment in each case

\begin{tabular}{|c|c|c|c|c|c|c|}
\hline Devices & 1 & 2 & 3 & 4 & 5 & 6 \\
\hline 0.035-inch Radifocus Standard ${ }^{\mathrm{a}}$ & 0 & 0 & 0 & 0 & 0 & O \\
\hline 0.035-inch Radifocus Stiffa & & & o & & & \\
\hline 0.035-inch Quick Flex Standard ${ }^{\mathrm{b}}$ & & & & & 0 & \\
\hline 0.010-0.014-inch Tenrou ${ }^{\mathrm{b}}$ & 0 & O & O & 0 & & ○ \\
\hline 0.014-inch CHIKAI ${ }^{\mathrm{c}}$ & & o & o & & O & o \\
\hline 0.010-inch X-Pedion ${ }^{d}$ & & & & & ○ & \\
\hline 0.010-inch SilverSpeed ${ }^{d}$ & & & & ○ & & \\
\hline 0.014-inch Traxcess ${ }^{\mathrm{e}}$ & & & & & & 0 \\
\hline Excelsior SL-10 ${ }^{f}$ & ○ & 0 & ○ & ○ & ○ & \\
\hline Headway $17^{e}$ & & & & O & & O \\
\hline Prowler Select Plus ${ }^{g}$ & & & & o & & \\
\hline Hydrogel coils ${ }^{\mathrm{e}}$ & 0 & & & & 0 & \\
\hline ED coils ${ }^{\mathrm{b}}$ & o & 0 & 0 & & o & \\
\hline Target coils ${ }^{f}$ & ○ & o & & & & O \\
\hline Cashmere $^{\mathrm{g}}$ & & ○ & & & & \\
\hline$V_{F C}$ & & 0 & & & & \\
\hline Orbit Galaxy ${ }^{g}$ & & ○ & & 0 & o & \\
\hline Deltapaq ${ }^{g}$ & & ○ & & & & \\
\hline Deltaplush ${ }^{g}$ & & o & & & & \\
\hline Matrix coils ${ }^{f}$ & & & & & 0 & \\
\hline Scepter XC & & & 0 & & & 0 \\
\hline HyperForm ${ }^{d}$ & & & & & 0 & \\
\hline Enterprise VRD ${ }^{g}$ & & & & 0 & & \\
\hline Amplatz GooseNeck Snare ${ }^{d}$ & & & & & ○ & \\
\hline
\end{tabular}

Note:- 0 indicates that the product was used.

${ }^{a}$ Terumo, Tokyo, Japan.

${ }^{\mathrm{b}}$ Kaneka Medix, Osaka, Japan

c Asahi Intecc, Aichi, Japan.

${ }^{d}$ Covidien, Irvine, California.

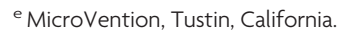

f Stryker, Kalamazoo, Michigan.

g Codman \& Shurtleff, Raynham, Massachusetts.

T2*-weighted images (Fig 1H). Head CT (Aquilion ONE CT scanner; Toshiba Medical Systems, Tokyo, Japan) obtained on the following day demonstrated no abnormal findings except a coil mass. The patient was discharged from our hospital 1 week after the coil embolization without any symptoms. Follow-up MR imaging was performed at 13 months after the procedure, and both abnormal spots remained without any signal changes (Fig $1 I,-L$ ). The patient remained asymptomatic at the last follow-up.

Case 5. A 70-year-old man was referred to our hospital for treatment of his unruptured anterior communicating artery aneurysm (Fig 2A). Preprocedural MR imaging showed no abnormal findings apart from the aneurysm. The coil embolization was performed; a $6 \mathrm{~F}$ guiding catheter was placed in his left ICA. After a balloon catheter was placed underneath the aneurysmal neck by using a 0.01 -inch microguidewire, a $1.7 \mathrm{~F}$ microcatheter was placed in the aneurysm by using another 0.014 -inch microguidewire. During the embolization, the first coil uncoiled, and it was withdrawn by using a retrieval device. Because the hub of the
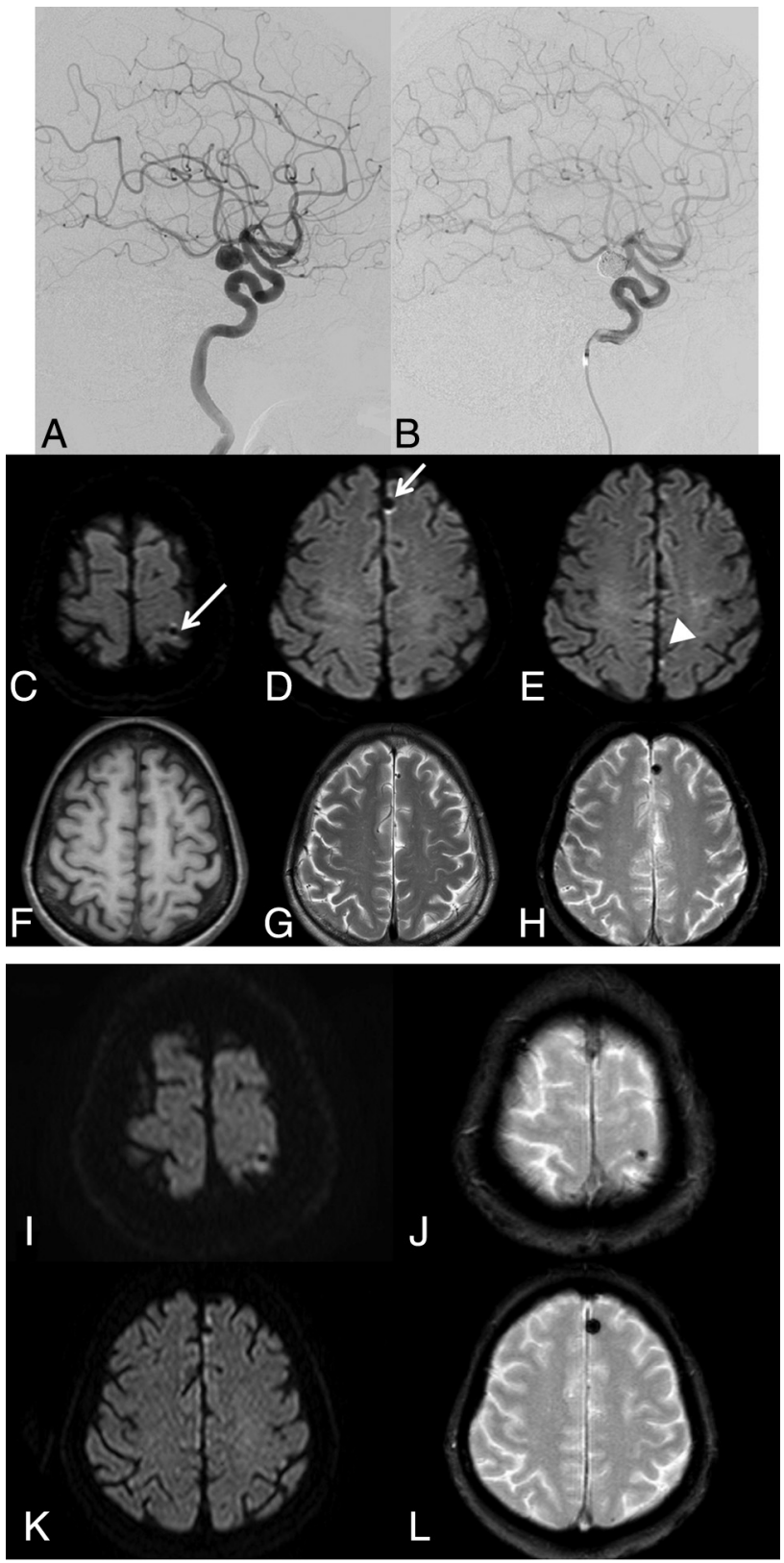

FIG 1. A left ICA aneurysm in case $1(A)$ is embolized by using multiple coils (B). Postprocedural DWI shows abnormal low-intensity spots with a hyperintense halo (white arrow) in the left parietal (C) and frontal ( $D$ ) lobes. These are different from a bright spot in the left parietal lobe $(E)$, indicating thromboembolism. These abnormal spots are low intensity on TIWI $(F)$ and T2WI $(G)$ and show a blooming effect on $\mathrm{T}^{*}$-weighted images $(H)$. Follow-up MR imaging obtained 13 months after the procedure shows that all the abnormal signal spots remain without any signal changes $(I-L)$.

AJNR Am J Neuroradiol 37:1696-99 Sep 2016 www.ajnr.org 
microcatheter had to be cut to advance the retrieval device, another $1.7 \mathrm{~F}$ microcatheter was used for the embolization. The aneurysm was finally embolized by using multiple platinum coils (Fig $2 B$ ). The MR imaging performed on postoperative day 1 by using a $1.5 \mathrm{~T}$ machine (Achieva Nova Dual; Philips) with 5-mm section thickness demonstrated a low-intensity spot with a hyperintense halo on DWI in the right frontal lobe (Fig 2C). A head CT scan (Aquilion ONE; Toshiba) with 5-mm section thickness obtained on postoperative day 3 showed no abnormal findings except a coil mass (Fig 2D). The patient's postoperative course was good, and he was discharged from our hospital 1 week after the procedure without any symptoms. Follow-up MR imaging obtained 3 years after the procedure demonstrated that the lesion remained at the right frontal lobe without any signal changes (Fig $2 E,-F$ ). The patient remained asymptomatic at the last follow-up.

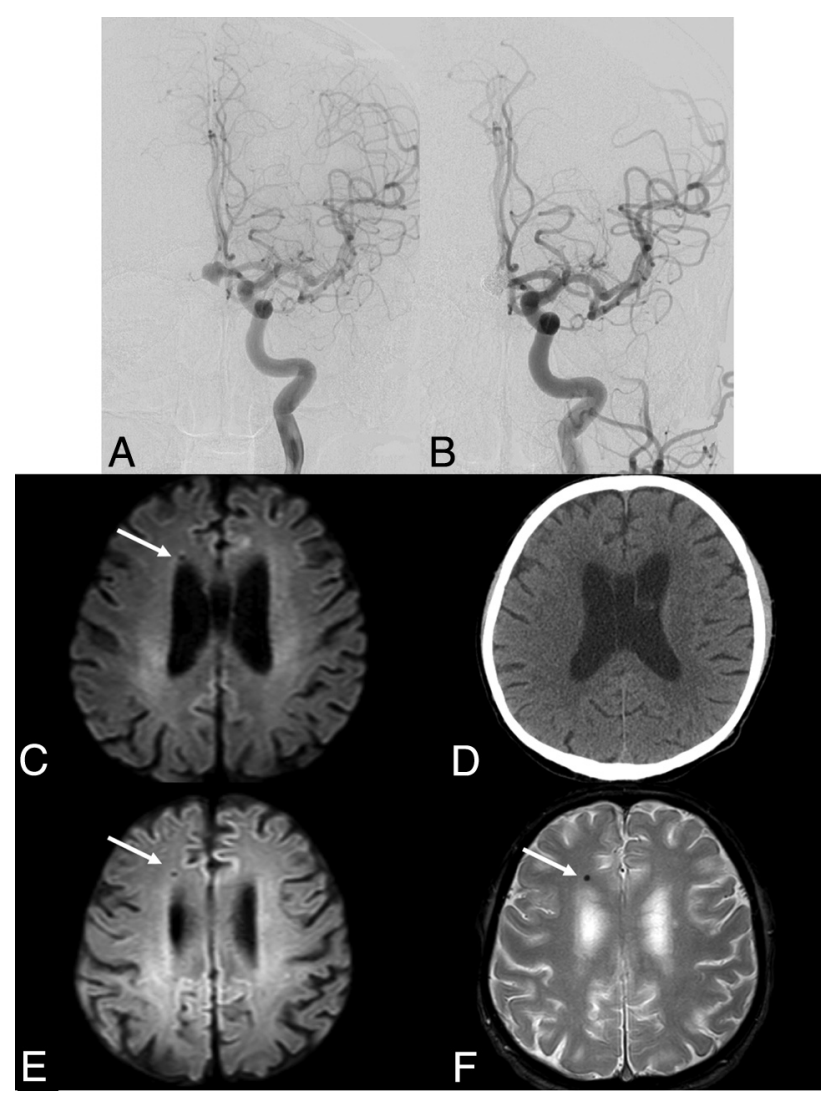

FIG 2. An anterior communicating artery aneurysm in case $5(A)$ is embolized by using multiple coils $(B)$. Postprocedural DWI shows an abnormal low-intensity spot with a hyperintense halo (white arrow) in the right frontal lobe $(C)$, which is undetectable on the postprocedural CT (D). Follow-up MR imaging obtained 3 years after the procedure shows that the lesion remains without any signal changes $(E$ and $F$ ).

\section{DISCUSSION}

Because the new low-signal spotty lesions appeared just after the endovascular procedure and they were restricted to the vascular territory of the catheterized arteries, it is reasonable to believe they represented embolic phenomena.

Potential etiologies of the low-signal spotty lesions include air, thrombus, hemosiderin, calcium deposits, and metal. If they were air or thrombus, resolution of signal changes would be expected with time. Moreover, parenchymal ischemic changes due to an embolism could occur. Hemosiderin is also unlikely because it does not appear at this early stage just after coil embolization. Furthermore, if the lesions were calcium, they might be detected by CT. Metal results in a dark spot on MR imaging because it has no MR imaging signal. Additionally, it can cause substantial MR imaging artifacts, ${ }^{5}$ which could exhibit the hyperintense halo. Because only microscopic metal fragments are required to produce MR imaging artifacts, ${ }^{6}$ it is not surprising that the metal fragments were invisible on CT. Moreover, if microscopic metal fragments were present, it would be reasonable that patients had no embolic complications. Taking these observations into consideration, we believe that the spotty lesions found on the postoperative MR imaging in our cases were from metallic fragments.

Cerebral MR imaging findings suggesting embolic metal fragments are rare, and only 4 cases have been reported (Table 3 ). ${ }^{7-10}$ In addition to a mechanical prosthetic cardiac valve and robotic surgery, angiographic guidewires used during cardiac catheterization were suspected as an embolic source in 2 cases. ${ }^{9,}{ }^{10}$ To the best of our knowledge, this cases series is the first in reporting MR imaging metallic signal following neuroendovascular treatment of intracranial aneurysms. Besides the guidewires, various devices (ie, coils, balloons, and stents) composed of metal are used in neuroendovascular procedures, which can provide more frequent opportunities for metallic embolism compared with cardiac catheterization.

Our study has several limitations. First and most important, it was not pathologically confirmed that the lesion represented metallic emboli. Because our patients were completely free from symptoms, it was not reasonable to perform a brain biopsy. Second, we cannot determine which devices and which parts could be a source of metallic emboli in our cases. In neuroendovascular treatment, metallic emboli can be released at any step, including delivery; deployment or withdrawal of the devices; friction between the devices; and detachment of the coils. Coil uncoiling and withdrawal of the uncoiled coil were likely responsible for metallic emboli in case 5. However, in the remainder of cases, coil embolization was completed uneventfully. Although MR imaging susceptibility artifacts vary depending on metals, ${ }^{11}$ these were not metal-specific and there is no way to differentiate various metals on MR imaging. We have reviewed all the devices used during

Table 3: Past cases of suspected metallic embolism

\begin{tabular}{|c|c|c|c|c|c|c|}
\hline Author & Patient/Sex & T2WI/Proton & T2*-Weighted Image & CT & Suspected Etiology & Symptom \\
\hline Wingerchuk et $\mathrm{al}^{7}$ & $35 / F$ & Low intensity halo & Low intensity blooming & Normal & Prosthetic cardiac valve & None \\
\hline Naumann et $\mathrm{al}^{8}$ & $66 / M$ & Low intensity halo & Not available & Not available & Prosthetic cardiac valve & Seizure \\
\hline Jassal et $\mathrm{al}^{9}$ & $55 / \mathrm{M}$ & Low intensity halo & Not available & Normal & Coronary guidewire & Headache \\
\hline Roshal et $\mathrm{al}^{10}$ & $55 / F$ & Low intensity & $\begin{array}{l}\text { Low intensity blooming, } \\
\text { halo }\end{array}$ & Normal & $\begin{array}{l}\text { Coronary guidewire, } \\
\text { robotic surgery }\end{array}$ & Headache \\
\hline
\end{tabular}


endovascular treatment in these cases (Table 3). No specific devices except one 0.035 -inch guidewire (0.035-inch Radifocus Standard; Terumo, Tokyo, Japan) were used in all cases. It is unlikely, however, that the 0.035 -inch guidewire is responsible because we have routinely used the guidewire in all endovascular procedures, including diagnostic angiography. Devices for intracranial use seem more likely than the 0.035 -inch guidewire. Further investigations should be done in the near future.

Finally, the exact incidence of the abnormal MR imaging signals was unclear. Our retrospective review included 110 coiling procedures for aneurysm treatment. Although periprocedural MR imaging was available in almost all cases, many cases lacked pre- and/or postoperative DWI, T2* -weighted images, or SWI, and only 29 cases had adequate imaging. Prospective registration with a specific perioperative MR imaging protocol is ongoing.

\section{CONCLUSIONS}

This is the first report on the brain MR imaging findings suggesting that metallic emboli were found after coil embolization of cerebral aneurysms, to our knowledge. Particular DWI and T2*weighted imaging findings of low intensity accompanied by a hyperintense halo should be kept in mind for patients undergoing coil embolization.

\section{REFERENCES}

1. Bendszus M, Koltzenburg M, Burger R, et al. Silent embolism in diagnostic cerebral angiography and neurointerventional procedures: a prospective study. Lancet 1999;354:1594-97 CrossRef Medline

2. Mehta RI, Mehta RI, Solis OE, et al. Hydrophilic polymer emboli: an under-recognized iatrogenic cause of ischemia and infarct. Mod Pathol 2010;23:921-30 CrossRef Medline

3. Cruz JP, Marotta T, O'Kelly C, et al. Enhancing brain lesions after endovascular treatment of aneurysms. AJNR Am J Neuroradiol 2014;35:1954-58 CrossRef Medline

4. Minks D, Briley D, Schulz U, et al. Suspected cerebral foreign body granuloma following endovascular treatment of intracranial aneurysm: imaging features. Neuroradiology 2015;57:71-73 CrossRef Medline

5. Hargreaves BA, Worters PW, Pauly KB, et al. Metal-induced artifacts in MRI. AJR Am J Roentgenol 2011;197:547-55 CrossRef Medline

6. Alanen A, Bondenstam S, Komu M. Artifacts in MR imaging caused by small quantities of powdered iron. Acta Radiol 1995;36:92-95 Medline

7. Wingerchuk DM, Krecke KN, Fulgham JR. Multifocal brain MRI artifacts secondary to embolic metal fragments. Neurology 1997;49: 1451-53 CrossRef Medline

8. Naumann M, Hofmann E, Toyka KV. Multifocal brain MRI hypointensities secondary to embolic metal fragments from a mechanical heart valve prosthesis: a possible source of epileptic seizures. $\mathrm{Neu}$ rology 1998;51:1766-67 CrossRef Medline

9. Jassal DS, Fast MD, McGinn G. Multifocal brain MRI hypointensities secondary to cardiac catheterization. Neurology 2000;54: 2023-24 CrossRef Medline

10. Roshal D, Snapp M, Friedman DP, et al. Iatrogenic brain and cervical cord magnetic resonance imaging susceptibility artifacts from metallic microemboli. Arch Neurol 2011;68:132-33 CrossRef Medline

11. Matsuura $\mathrm{H}$, Inoue $\mathrm{T}$, Konno $\mathrm{H}$, et al. Quantification of susceptibility artifacts produced on high-field magnetic resonance images by various biomaterials used for neurosurgical implants: technical note. J Neurosurg 2002;97:1472-75 CrossRef Medline 